\title{
AMPLIACIÓN DEL ÁREA DE DISTRIBUCIÓN GEOGRÁFICA DE OXYBELIS FULGIDUS (SERPENTES: COLUBRIDAE) Y AGKISTRODON BILINEATUS (SERPENTES: VIPERIDAE) EN LA PLANICIE COSTERA CENTRAL DE OAXACA, MÉXICO
}

García-Grajales, J. \& A. Buenrostro-Silva. 2011. Geographic distribution enlargment of Oxybelis fulgidus (Serpentes: Colubridae) and Agkistrodon bilineatus (Serpentes: Viperidae) from the costal central plain of Oaxaca, Mexico. Acta Zoológica Mexicana (n. s.), 27(2): 491-495.

ABSTRACT. This report indicates the presence of Oxybelis fulgidus and Agkistrodon bilineatus in Parque Nacional Lagunas de Chacahua, Oaxaca, extending their distribution from its known range to 286 and $343 \mathrm{~km} \mathrm{NW}$ respectively.

La herpetofauna de Oaxaca ha sido estudiada parcialmente bajo un contexto macrofaunístico (Casas-Andreu et al. 2004) aunque la narración histórica de la contribución al conocimiento de los anfibios y reptiles del estado ha sido ampliamente documentada (Flores-Villela 1993, Casas-Andreu 1996); también se ha realizado y actualizado una primera síntesis de las especies herpetofaunísticas conocidas en la entidad (Casas-Andreu et al. 1996, 2004). No obstante, siguen existiendo huecos geográficos con posibilidades de generar nuevos registros y ampliaciones de la distribución de las especies de anfibios y reptiles documentadas para este estado.

En el grupo de las serpientes, la familia Colubridae es una de las más diversas distribuyéndose en todo el mundo a excepción de la Antártida y las islas oceánicas (Zug et al. 2001, Köhler 2003). En México, es la familia con el mayor número de géneros y especies (Liner 2007). Específicamente, en el género Oxybelis se encuentran organismos comúnmente denominados como "bejuquillos", por su cabeza notablemente alargada y acuminada hacia el rostro, sus cuerpos largos y delgados y su colorido muy semejante al del medio en que se encuentran y debido a sus hábitos predominantemente arborícolas pasan inadvertidos entre el follaje (Campbell 1998, Lee 2000, 
Köhler 2003, Pérez-Higareda et al. 2007). Los registros históricos más norteños de la distribución de Oxybelis fulgidus corresponden a las inmediaciones del Istmo de Tehuantepec en la localidad de Santo Domingo (Daudin 1803 en Köhler 2003). De las localidades de Chihuitán y Tres Cruces se colectaron y depositaron ejemplares de esta especie en 1940 en el Departamento de Zoología de Vertebrados del Smithsonian National Museum of Natural History (USNM-110561 y 110563). Por otra parte, en el grupo de las serpientes venenosas de la familia Viperidae, Agkistrodon bilineatus presenta una distribución en parches a lo largo de la vertiente del Pacífico así como en la península de Yucatán (Lee \& Hammerson 2007). Su distribución en Oaxaca se conoce únicamente en las inmediaciones del Istmo de Tehuantepec (Köhler 2003, Lee \& Hammerson 2007) (Fig. 1). Es una serpiente mediana de hábitos terrestres y nocturnos que habita en áreas cercanas a cuerpos de agua (Lee 2000, Köhler 2003).

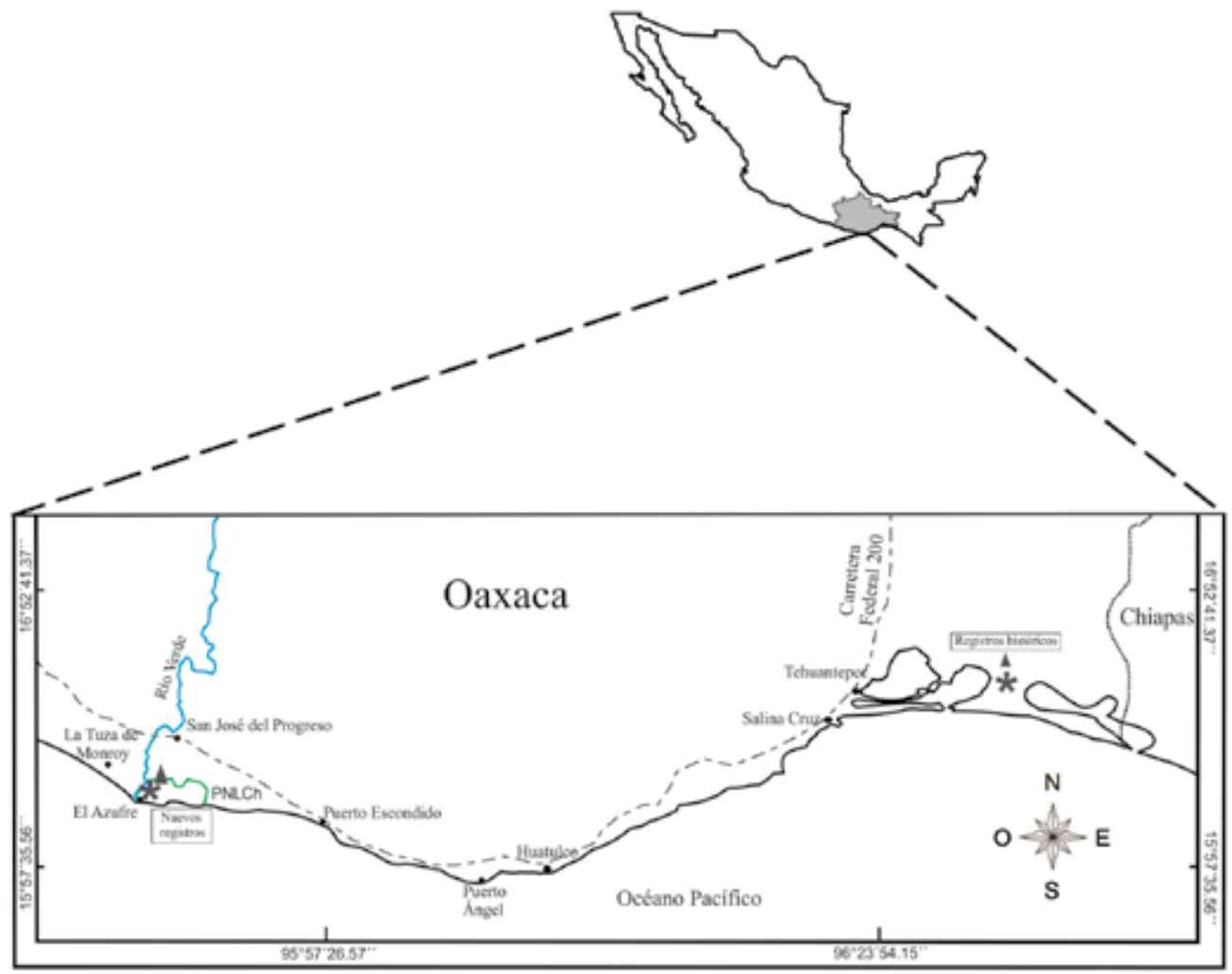

Figura 1. Localización de los registros actuales e históricos para Oxybelis fulgidus (*) y Agkistrodon bilineatus ( $\boldsymbol{\Delta}$ ) dentro del Parque Nacional Lagunas de Chacahua (PNLCh) en la planicie costera de Oaxaca (basado en Lee \& Hammerson 2007). 
En este trabajo se aporta información adicional sobre la distribución de $O$. fulgidus y A. bilineatus con base en registros obtenidos a partir de recorridos y muestreos sistemáticos entre febrero de 2009 y febrero de 2010 en la Cuenca Baja del Río Verde, Oaxaca. Esta área está comprendida desde la localidad de La Tuza de Monroy en el municipio de Santiago Jamiltepec hasta la localidad de Cerro Hermoso en el municipio de San Pedro Tututepec, incluye el polígono del Parque Nacional Lagunas de Chacahua y se ubica en la Región Terrestre Prioritaria (RTP) 128: Bajo Río Verde Chacahua.

El 2 de septiembre de 2009 a las $1520 \mathrm{~h}$, en el camino de terracería principal que conduce de San José del Progreso a la comunidad de Chacahua y dentro del polígono del Parque Nacional Lagunas de Chacahua, en el municipio de San Pedro Tututepec, se capturó a una hembra adulta de O. fulgidus (Fig. 2A) que cruzaba el camino, aproximadamente a $1.2 \mathrm{~km}$ de la comunidad de El Azufre, municipio de San Pedro Tututepec, en la Cuenca Baja del Río Verde (UTM 14Q 0631945 E, 1771022 N, 8 $\mathrm{msnm})$. Se decidió no colectar al organismo debido a que probablemente la abundan-

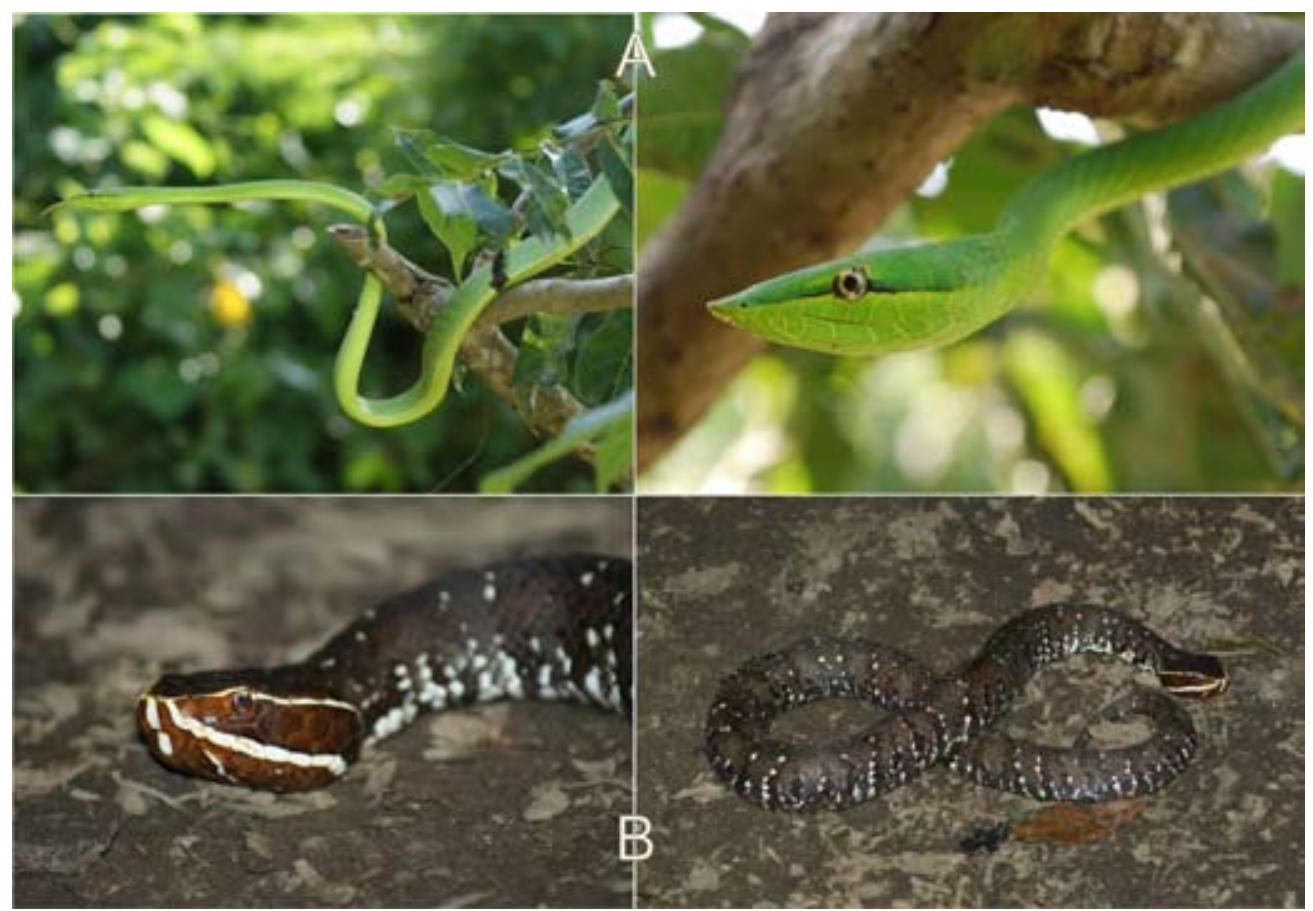

Figura 2. Registro fotográfico de ejemplares en vida silvestre de (A) Oxybelis fulgidus y (B) Agkistrodon bilineatus encontrados en el Parque Nacional Lagunas de Chacahua, Oaxaca. 
cia de esta especie es baja y porque un individuo hembra representa para la especie la oportunidad de asegurar su persistencia en la zona. Únicamente se obtuvieron registros fotográficos del ejemplar y se depositaron en la colección fotográfica del Laboratorio de Herpetología de la Universidad del Mar campus Puerto Escondido con números de catálogo 0031 y 032 . Este registro extiende el área de distribución de $O$. fulgidus, aproximadamente $286 \mathrm{~km}$ (en línea recta) al NO de la localidad más norteña de su distribución en la costa de Oaxaca.

Por otra parte, el 3 de septiembre de 2009 a las 22:45 h, en el paraje denominado "La Tranca" dentro del Parque Nacional Lagunas de Chacahua, se observó y capturó a un individuo adulto de A. bilineatus a $900 \mathrm{~m}$ de la comunidad de El Azufre (UTM 14 Q 0632535 E, 1771389 N, 8 msnm), municipio de San Pedro Tututepec (Fig. 2B). Se obtuvieron registros fotográficos del individuo y se depositaron en la colección fotográfica del Laboratorio de Herpetología de la Universidad del Mar campus Puerto Escondido con números de catálogo 0038 y 0039. Este registro extiende el área de distribución de $A$. bilineatus, aproximadamente $343 \mathrm{~km}$ (en línea recta) al NO de Tres Cruces Tehuantepec, la localidad más cercana previamente registrada en esta región (Günther 1863 en Köhler 2003).

Estos dos nuevos registros contribuyen a actualizar el inventario de reptiles en la planicie costera de Oaxaca. Siendo el orden Serpentes el que tiene mayor representatividad nacional, la continuidad de los inventarios herpetofaunísticos en esta zona enriquecerá el conocimiento sobre la distribución de estos vertebrados en la región.

AGRADECIMIENTOS. Agradecemos a la Universidad del Mar (UMAR) el financiamiento otorgado a través del convenio CFE-UMAR (CUP 2IR0807). También agradecemos de manera muy especial a los pobladores de El Azufre por las facilidades prestadas. Dos revisores anónimos contribuyeron a enriquecer el contenido del manuscrito.

\section{LITERATURA CITADA}

Campbell, J. 1998. Amphibians and reptiles of Northern Guatemala, the Yucatan and Belize. University of Oklahoma Press. Oklahoma.

Casas-Andreu, G. 1996. Notas para la historia de los estudios herpetofaunísticos en el estado de Oaxaca, México. Boletín Sociedad Herpetológica Mexicana 7(1): 21-26.

Casas-Andreu, G., F. R. Méndez-De la Cruz \& J. L. Camarillo. 1996. Anfibios y reptiles de Oaxaca. Lista, distribución y conservación. Acta Zoológica Mexicana (nueva serie) 69: 1-35.

Casas-Andreu, G., F. R. Méndez-De la Cruz \& X. Aguilar-Miguel. 2004. Anfibios y reptiles, pp. 375-390. In: A. J. García-Méndoza, M. J. Ordoñez y M. Briones-Salas (Eds.). Biodiversidad de Oaxaca. Instituto de Biología, UNAM-Fondo Oaxaqueño para la Conservación de la NaturalezaWorld Wildlife Fund, México.

Flores-Villela, O. 1993. Breve historia de la herpetofauna en México. Elementos, 18: 11-21.

Köhler, G. 2003. Reptiles of Central America. Herpeton Verlag. Offenbach.

Lee, J. C. 2000. A field guide to the amphibians and reptiles of the Maya World. The lowlands of Mexico, Northern Guatemala and Belize. Cornell University Press, Ithaca. 
Lee, J. \& G. A. Hammerson. 2007. Agkistrodon bilineatus. In: IUCN 2010. IUCN Red List of Threatened Species. Version 2010.2. <www.iucnredlist.org>. Consultado el 10 de agosto de 2010.

Liner, E. A. 2007. A checklist of the amphibians and reptiles of Mexico. Louisiana State University Occasional Papers of the Museum of Natural Science, 80: 1-60.

Pérez-Higareda, G., M. A. López-Luna \& H. M. Smith. 2007. Serpientes de la región de los Tuxtlas, Veracruz, México. Guía de identificación ilustrada. Universidad Nacional Autónoma de México, México D. F.

Zug, G. R., L. J. Vitt \& J. P. Caldwell. 2001. Herpetology. An introductory biology of amphibians and reptiles. 2nd Edition. Academic Press, San Diego.

\section{Jesús García-Grajales ${ }^{1} \boldsymbol{\&}$ Alejandra Buenrostro-Silva ${ }^{2}$}

${ }^{1}$ Instituto de Recursos, Universidad del Mar, campus Puerto Escondido, Km 2.5, carretera a Sola de Vega, San Pedro Mixtepec 71980, Oaxaca, México.

$<$ archosaurio@yahoo.com.mx>

${ }^{2}$ Instituto de Industrias, Universidad del Mar, campus Puerto Escondido, Km 2.5, carretera a Sola de Vega, San Pedro Mixtepec 71980, Oaxaca, México. 